\title{
Produtos de autocuidado para clareamento dental
}

\author{
Self-care products for tooth whitening
}

\author{
Flávio Fernando Demarco* \\ Ana Carolina Gluszevicz** \\ Maria Luiza M. Mendes* \\ Mariana M. C. do Nascimento* \\ Natália Silveira Cabreira* \\ Valentina Crugeira Barbieri**
}

\section{Resumo}

Objetivo: fazer uma revisão da literatura sobre os vários métodos de produtos de autocuidado para clareamento dental, procurando avaliar as evidências em relação à sua efetividade e às limitações. Revisão de literatura: a preocupação com a estética dental tem ganhado importância nos últimos anos, o clareamento dental caseiro tem sido um tratamento cada vez mais procurado nos consultórios odontológicos e o tratamento caseiro com moldeiras contendo agentes clareadores tem apresentado resultados satisfatórios, com poucos efeitos adversos, sendo considerado o padrão-ouro para tratar dentes vitais. Com o sucesso dessa técnica, novos produtos, conhecidos como de autocuidado para clareamento dental, têm aparecido no mercado, os quais são livremente comercializados e podem ser utilizados sem a supervisão do dentista. Diferentes produtos podem ser encontrados dentro dessa classificação (fitas clareadoras, dentifrícios clareadores, enxaguatórios, vernizes), mas o período relativamente recente de sua introdução e a pequena quantidade de estudos clínicos realizados geram dúvidas acerca de sua efetividade e sobre potenciais efeitos adversos. A fita adesiva apresentou um resultado satisfatório em algumas pesquisas, além de ser prática e simples para o próprio uso. Considerações finais: diante dos estudos realizados com base na eficácia dos produtos de autocuidado para clareamento dental e com base na revisão de literatura, nota-se que esses não substituem um tratamento clareador convencional em consultório, visto que a maioria não cumpre sua ação clareadora.

Palavras-chave: Clareamento dental. Cor. Dentifrício.

\section{Introdução}

A preocupação das pessoas com a estética cresceu com o passar dos anos, inclusive com a aparência dental, uma vez que ter dentes brancos e alinhados é considerado fator importante de aceitação e elevação da autoestima. Dessa forma, o sorriso tem sido fundamental na sociedade, sendo que sua excelência constitui o padrão de beleza ideal ${ }^{1}$.

Dentre os métodos utilizados para a recuperação da descoloração dentária, o clareamento dental é o mais conservador. O clareamento pode ser realizado no consultório odontológico ou em casa, sob a supervisão do dentista, com agentes clareadores colocados em moldeiras ou com produtos clareadores usados pelo paciente, sem supervisão profissional ${ }^{2}$. O clareamento caseiro com peróxido de carbamida (PC) a $10 \%$ tem sido um dos procedimentos mais requisitados em odontologia e é considerado o gold standard em termos de efeito clareador e baixo nível de efeitos adversos para clareamento de dentes vitais ${ }^{3}$.

Tendo em vista a popularidade e os resultados estéticos favoráveis do tratamento clareador caseiro com PC 10\%, ocorreu um aparecimento de novos produtos no mercado, os quais apresentam diferentes formas de uso, contendo ou não agentes clareadores, e que podem ser comprados livremente em farmácias, supermercados ou pela internet, sendo utilizados sem a supervisão do odontólogo. Esses produtos, chamados de autocuidado ou OTC (do in-

\footnotetext{
Professor Associado do Departamento de Dentística, Faculdade de Odontologia, Universidade Federal de Pelotas, Pelotas, Rio Grande do Sul, Brasil.

Graduandas em Odontologia, Faculdade de Odontologia, Universidade Federal de Pelotas, Pelotas, Rio Grande do Sul, Brasil.
} 
glês over-the-counter), foram introduzidos nos Estados Unidos por volta do ano 2000, como alternativa mais econômica em relação ao tratamento realizado pelos dentistas no consultório ou com o uso de mol$\operatorname{deiras}^{2,4}$.

O presente trabalho tem como objetivo realizar uma revisão de literatura sobre os diferentes produtos de autocuidado para clareamento dental, procurando discutir sua efetividade e os potenciais problemas relacionados com o seu uso.

\section{Revisão de literatura}

\section{Dentifrícios clareadores: verdades e mitos}

Inúmeros dentifrícios têm sido expostos pela mídia como a chave para o sorriso esteticamente perfeito, cujas propagandas asseguram o clareamento dos dentes baseando-se na composição dessas substâncias, que, normalmente, é representada pelos peróxidos, como o de carbamida, o de hidrogênio ou o de cálcio ${ }^{5}$. O aumento da procura por esses dentifrícios clareadores pode ser entendido pelo fato de aparentar ser uma forma mais simples de se livrar de manchas desagradáveis ${ }^{6}$.

Surgiram hipóteses para tentar explicar os efeitos que os fabricantes afirmavam ter seus respectivos produtos, mas na maioria desses, o efeito é quase nulo, ou seja, a ação clareadora por meio de dentifrícios clareadores é praticamente inexistente. Essa eficiência limitada pode ser explicada tendo em vista que muitos desses produtos contêm apenas abrasivos para remoção de manchas extrínsecas da superfície dental ${ }^{7}$. Essas manchas extrínsecas têm causas variadas, como fumo e hábitos alimentares em que há excesso de ingestão de substâncias corantes, tais como chá, café, entre outras ${ }^{8}$.

O clareamento dental a partir do peróxido é um método já estabelecido, porém, a utilização desse no dentifrício é limitada, devido às alterações na fórmula e à necessidade de um tempo de contato prolongado, que não é possível apenas com o uso do dentifrício ${ }^{9}$. Logo, a mínima concentração dos peróxidos nessas substâncias e o curto tempo de contato dessas com os dentes resultam em uma função auxiliar, facilitando a ação dos abrasivos sobre as manchas extrínsecas. Os abrasivos são eficazes para a remoção dessas manchas, promovendo ainda o polimento dentário, os mais comuns são carbonato de cálcio, polímero acrílico, fosfato de cálcio, bicarbonato de sódio, sílica hidratada e metafosfato de sódio insolúvel ${ }^{10}$.

Mello ${ }^{11}$ (2014) avaliou in vitro o poder clareador dos cremes dentais Sensodyne - Branqueador, Colgate Total 12 Professional White, Sorriso-xtreme white, Close-Up-Extra Whitening, Oral B - Pró-Saúde Whitening, Colgate-Luminous White, Crest 3D White e Aquafresh Extreme Clean Whitening
Action, analisando-os quanto à eficácia na remoção de manchas extrínsecas, sendo o Oral B - Pró-Saúde Whitening, o Colgate-Luminous White e o Aquafresh Extreme Clean Whitening Action os que apresentaram melhor efeito de branqueamento. Em contrapartida, Silva et al. ${ }^{7}$ (2011) avaliaram o efeito dos cremes dentais clareadores Colgate Branqueadora, Malvatricin Branqueadora e creme dental clareador manipulado - Phormula Ativa, associados ou não à escova dental especial, concluindo que os cremes dentais não foram eficazes em produzir um efeito clareador significativo.

Dessa forma, é indispensável que o consumidor não se restrinja apenas ao que é exposto nos meios de comunicação, mas, sim, busque em pesquisas e trabalhos científicos relevantes as respostas para os seus anseios e dúvidas, já que os anúncios têm como objetivo a venda em massa, expondo apenas supostos benefícios ${ }^{6}$.

\section{Fita clareadora}

A procura pela fita clareadora para realizar o clareamento dental tem sido cada vez maior, porém, o consumidor nem sempre tem um real conhecimento sobre a forma correta da utilização, uma vez que essas podem ser aplicadas pelo próprio usuário sem acompanhamento profissional ${ }^{12}$. Por conseguinte, muitas pessoas acabam utilizando de forma incorreta, acreditando que com o uso prolongado e várias vezes ao dia obterão resultados melhores, no entanto, a fita clareadora acaba gerando sensibilidade dental acima do esperado ${ }^{13}$.

Em relação ao uso das fitas, cobre-se os seis dentes anteriores e aplica-se a fita de forma pressionada nesses, alinhando a extremidade superior à gengiva marginal dos dentes anteriores superiores, e na dentição inferior alinhando a longa superfície trapezoidal em relação à margem gengival dos dentes anteriores ${ }^{14}$. Mais comumente, essa fita é constituída à base de peróxido de hidrogênio ou peróxido de carbamida ${ }^{15}$.

No Brasil, a fita clareadora mais conhecida é a Oral-B 3D Whitestrips, de acordo com o fabricante, os resultados podem ser percebidos em três dias de uso, sendo usada durante uma semana, duas vezes ao dia, por trinta minutos ${ }^{16}$. As concentrações são variáveis e podem influenciar no número de dias de aplicação, desse modo, as Whitestrips (ORAL-B), com 5,3\% de concentração, devem ser utilizadas duas vezes ao dia por 14 dias, a Professional Whitestrips, que possui $6,5 \%$ de concentração, deve ser utilizada duas vezes ao dia durante 21 dias, e a Whitestrips Supreme, com $14 \%$ de concentração, deve ser usada uma vez por dia durante sete dias ${ }^{17}$. Já as fitas clareadoras Crest Whitestrips e Crest Professional Whitestrips contêm 5,3\% e 6,5\% de peróxido de hidrogênio, respectivamente, são utiliza- 
das duas vezes ao dia, por 30 minutos, durante duas semanas ${ }^{18}$.

De acordo com resolução da Agência Nacional de Vigilância Sanitária (Anvisa), os clareadores dentais somente são vendidos com prescrição do odontólogo ${ }^{19}$. Conforme a resolução, a determinação da Anvisa vale para produtos com concentrações de peróxido de carbamida e peróxido de hidrogênio superiores a 3\%, incluindo nessa proposta as fitas clareadoras. Entretanto, muitas vezes é possível a compra do produto pelo consumidor devido à negligência de alguns fornecedores ${ }^{20}$.

As fitas adesivas clareadoras têm mostrado, em estudos clínicos de curta duração, um efeito clareador similar ao produzido com o clareamento caseiro com o uso de moldeiras e peróxido de carbamida a $10 \%^{21}$. No entanto, muitos desses estudos foram patrocinados pelos fabricantes dos produtos, sendo necessário estudos independentes.

Segundo Consolaro ${ }^{22}$ (2013), as fitas clareadoras apresentam algumas limitações, como o fato de que o peróxido de hidrogênio pode entrar em contato com a mucosa bucal, de o usuário não poder controlar a frequência e o tempo de uso, assim como as condições da mucosa e dos dentes, a fim de receber o agente clareador. Como efeito adverso, as fitas podem produzir irritação gengival, especialmente quando mal empregadas, ou sensibilidade dentária, sobretudo no emprego de concentrações mais elevadas ou na utilização por tempo maior do que o recomendado ${ }^{2}$.

É importante destacar que, dentre todos os produtos de autocuidado para clareamento dental, as fitas clareadoras são as mais efetivas, porém, só se observa resultado positivo em dentes com pequeno grau de escurecimento ${ }^{23}$.

\section{Enxaguante bucal}

Os enxaguatórios bucais têm sido utilizados como produtos auxiliares na higiene bucal, visando reduzir a quantidade de placa e melhorar o hálito. Mais recentemente, apareceram no mercado enxaguatórios contendo em sua composição agentes clareadores, como o peróxido de hidrogênio a 1,5\%, os quais poderiam ser utilizados para proporcionar efeito clareador nos dentes, aliado ao efeito auxiliar na higiene bucal ${ }^{24}$.

A popularização desses enxaguatórios como produto de clareamento tipo OTC (over-the-counter) ocorreu devido à sua fácil aplicação, ao baixo custo e à ampla disponibilidade em supermercados e farmácias. Esses produtos, geralmente, contêm em sua formulação baixas concentrações de peróxido de hidrogênio, de $1 \%$ a $2 \%{ }^{2,24}$.

De acordo com Lima et al. ${ }^{24}$ (2012), o uso de enxaguante contendo peróxido de hidrogênio com concentração de 1,5\%, realizando-se duas aplicações durante o dia com duração de um minuto, em um período de 45 dias, apresenta redução na luminosidade dental, resultando em dentes mais opacos. Para os autores, a provável razão para essa diminuição na luminosidade foi a desmineralização do esmalte superficial causada por um $\mathrm{pH}$ baixo, em torno de 3,4 .

Dentre os enxaguatórios bucais mais conhecidos no Brasil estão o enxaguante Bucal Colgate Luminous White, o Listerine Whitening Pré-escovação, com aplicação de um minuto duas vezes ao dia, apresentando resultados em seis semanas de uso, e o Antisséptico Bucal Oral-B 3D White, que auxilia na remoção do tártaro, evitando que se acumule na superfície dental e provoque manchas ${ }^{25-27}$.

$\mathrm{O}$ uso de enxaguatórios à base de peróxido de hidrogênio tem demonstrado boa capacidade de remoção de manchas tanto in vitro como in vivo, tendo sua ação potencializada se utilizado juntamente à clorexidina (CHX), conforme observado em estudo clínico com 99 indivíduos submetidos à utilização de enxaguatórios bucais. Ao final do estudo, mostrou-se muito satisfatório na redução da placa e na prevenção do desenvolvimento de manchas ${ }^{28}$.

Hasturk et al. ${ }^{29}$ (2004) verificaram a eficácia de um enxaguatório bucal à base de peróxido de hidrogênio fluoretado no clareamento dental e no tratamento de gengivite comparado a um placebo, os autores observaram que o produto testado foi considerado seguro durante um período de seis meses, verificando-se efeito clareador e redução na ocorrência de gengivite.

Durante o estudo de Torres et al..$^{30}$ (2013), foram analisados os efeitos da utilização dos enxaguatórios bucais Listerine Whitening e Colgate Plax Whitening, ambos contendo concentrações de peróxido de hidrogênio. Os resultados assemelharam-se aos observados após catorze dias com tratamento à base de peróxido de carbamida com concentração de $10 \%$. Todavia, as concentrações de peróxido nesses produtos são muito pequenas, a ponto de seu potencial clareador ser muito questionável, não expressando grande relevância clínica. $\mathrm{O}$ que torna preocupante a sua utilização é o fato de serem empregados sem a devida orientação e sem o acompanhamento profissional ${ }^{1}$.

Silva $^{31}$ (2013) avaliou a microdureza do esmalte após a utilização de agentes clareadores, sendo que o enxaguatório bucal Listerine Whitening Pré-escovação demonstrou ser um agente seguro sem estar associado ao dentifrício abrasivo e na presença do gel clareador, mantendo os níveis de dureza inalterados durante todo o experimento. Em contrapartida, conforme Consolaro et al..$^{32}$ (2011), agentes clareadores à base de peróxido de hidrogênio agem como ácidos na superfície do esmalte, podendo torná-lo poroso e gerar necrose nos tecidos moles. Assim, o consumo indiscriminado de bochechos com produtos clareadores pode não oferecer segurança de uso e deve ser supervisionado por um profissional.

Torna-se importante observar e avaliar o efeito de enxaguatórios bucais com presença de agente 
clareador na estrutura do esmalte dental, baseando-se em evidências científicas e critérios éticos que permitam ao odontólogo uma escolha correta, relacionada à segurança biológica e à verdadeira relação custo-benefício do que está sendo empregado ${ }^{33}$.

\section{Paint-on gels (vernizes)}

Os vernizes (Paint-on gels) são produtos de clareamento de autocuidado sem barreiras que apresentam em sua constituição os agentes peróxido de hidrogênio ou carbamida em diferentes concentrações. A exemplo de alguns vernizes encontrados no mercado, como Simply White e Simply White Night, contendo, respectivamente, $18 \%$ de peróxido de carbamida e $8,7 \%$ de peróxido de hidrogênio, fabricados pela Colgate, MyXantia com peróxido de carbamida, fabricado pela Dexcel Pharma, e Crest Night Effects, com $19 \%$ de percabonato de sódio, fabricado por Procter \& Gamble ${ }^{15}$.

De acordo com Slezak ${ }^{34}$ (2002) e Maia et al..$^{35}$ (2005), o produto de uso noturno de peróxido de hidrogênio a $8,78 \%$ e peróxido de carbamida a $30 \%$ deve ser usado uma vez ao dia, por um período de 6 $\mathrm{h}$ a $8 \mathrm{~h}$, por duas semanas, enquanto o produto de uso diurno à base de peróxido de hidrogênio a 5,4\% e peróxido de carbamida a $18 \%$ tem indicação de uso de duas vezes ao dia, por 30 minutos, durante 21 dias. Segundo Brunato ${ }^{18}$ (2005), o produto deve ser aplicado após a escovação em superfície dentária seca, com uma camada desse em um dente por vez, evitando que a língua e os lábios toquem os dentes por 30 segundos, sem comer ou beber durante os 30 minutos necessários para o clareamento.

Em ensaio clínico randomizado, Nathoo et al..$^{36}$ (2002) constataram que os dentes dos indivíduos do grupo tratado com o verniz contendo $18 \%$ de peróxido de carbamida, Colgate Simplesmente White Clear Whitening Gel, apresentaram melhora em comparação com os dentes no grupo placebo gel, clareando os dentes significativamente. Todavia, Gerlach et al..$^{37}$ (2004) avaliou a eficácia de três produtos, paint-on gel com $18 \%$ de peróxido de carbamida, creme dental clareador e as tiras de clareamento com $6 \%$ de peróxido de hidrogênio e verificou que não houve diferenças significativas na melhoria da cor para o paint-on gel em comparação com a escovação normal com um creme dental clareador, e que as tiras clareadoras resultaram em melhora superior na cor do dente. Esses resultados demonstram a controvérsia em relação à efetividade desse tipo de clareador OTC.

Esses produtos não apresentam o uso de barreiras, oferecendo conforto e fácil aplicação, contudo, se forem usados sem supervisão podem provocar o contato de substâncias cancerígenas com os tecidos moles. Ainda, o produto fica em contato com a saliva, podendo ser ingerido e, em indivíduos que o utilizam com frequência, gerar dor de estômago, sendo necessário determinar como esses sistemas podem afetar os tecidos do corpo ${ }^{38}$.

\section{Venda livre de clareadores dentais na internet}

Atualmente, as redes de sites de compra pela internet exercem grande influência sobre os consumidores dos mais diversos produtos, que podem ser ofertados em condições e preços cada vez mais acessíveis, colocando em dúvida sua qualidade e procedência. Os produtos de clareamento dental são facilmente encontrados em sites de vendas pela internet, uma vez que, no Brasil, não há venda restrita, desde que sejam registrados pela Anvisa, motivando sua proliferação em larga escala por meio da ampla rede de acesso pelos usuários ${ }^{39}$.

Contudo, de acordo com resolução da Anvisa, os clareadores dentais com concentrações de peróxido de carbamida e peróxido de hidrogênio superiores a 3\% somente serão vendidos com prescrição do odontólogo ${ }^{19}$. Em vista disso, conforme a Lei $\mathrm{n}^{\mathrm{o}} \quad 6.360 / 1976^{40}$, a propaganda de medicamentos ou produtos com exigência de venda sob prescrição odontológica ficará restrita a publicações destinadas à distribuição a cirurgiões-dentistas.

De acordo com o estudo de Oliveira et al. ${ }^{41}$ (2014), na Região Sudeste, houve a maior quantidade de ofertas em sites de compras coletivas. Ainda, o serviço ou produto mais promovido e ofertado foi o clareamento dental, remetendo à preocupação do público consumidor com a estética bucal.

Dentre os produtos mais encontrados nesse mercado, estão: os cremes dentais Sensodyne Branqueador, Colgate Total 12 Professional White, Close-Up-Extra Whitening, Oral B - Pró-Saúde Whitening e Colgate Luminous White ${ }^{42,43}$; A fita clareadora Oral-B 3D White Whitestrips ${ }^{44}$; enxaguatórios bucais clareadores Listerine Whitening Pré-escovação, Enxaguante Bucal Colgate Luminous White e Antisséptico Bucal Oral-B 3D White ${ }^{25-27}$; Paint-ongels vernizes Simply White e Simply White Night, da Colgate, e Crest Night Effects, fabricado por Procter \& Gamble ${ }^{45,46}$.

Alguns fatores têm sido determinantes para a efetivação da compra desses produtos, um deles é o baixo custo de aquisição associado à facilitação promovida por sites de compras coletivas e empresas de itens odontológicos. Ao visar, apenas, ao lucro, muitos estabelecimentos têm ignorado as precauções necessárias quanto ao uso indiscriminado do produto pelos compradores, o que pode acarretar muitos riscos à saúde, pois não há diagnóstico nem orientação de um profissional da área que administre as concentrações variadas do peróxido de carbamida, por exemplo, de acordo com as necessidades pontuais de cada paciente. Isso fere o código de defesa do consumidor ${ }^{47}$, além de criar um embate com os preceitos éticos da Odontologia e com a Lei 
$\mathrm{n}^{\mathrm{o}} 5.081 / 1966^{48}$, que dispõe sobre o exercício profissional no país, conforme cita o Conselho Federal de Odontologia ${ }^{49}$.

\section{Discussão}

Os estudos analisados são unânimes em ressaltar que os cremes dentais clareadores não produzem um clareamento expressivo, contendo abrasivos que atuam na superfície dentária, promovendo um polimento dentário, tendo eficácia restrita à remoção de manchas extrínsecas ${ }^{7,11,36,50}$.

Dentre os estudos que avaliaram as fitas clareadoras, encontraram-se resultados satisfatórios com efeito clareador semelhante ao clareamento com o uso de moldeiras e peróxido de carbamida a $10 \%$, concluindo-se, também, pela praticidade e simplicidade dessas, entretanto, não se sabe se as fitas têm a mesma durabilidade e o prognóstico do tratamento com moldeiras. Além disso, elas somente podem ser vendidas com prescrição do profissional e usadas sob a supervisão desse ${ }^{19,21}$.

Torres et al. ${ }^{30}$ (2013) e Hasturk et al. ${ }^{29}$ (2004) relataram que os enxaguatórios bucais à base de peróxido de hidrogênio conferem um efeito clareador. Em contrapartida, alguns autores destacam apenas a prevenção do desenvolvimento de manchas, sendo que o clareamento não expressa relevância clínica $^{1,27,28}$. Da mesma forma, os produtos Paint-on gels, observados pelo número de ensaios clínicos disponíveis, apresentam baixos níveis de agentes clareadores, podendo produzir um clareamento que não é clinicamente significativo ${ }^{2}$.

Um fator de risco em relação aos produtos de autocuidado é a sua venda exacerbada e sem controle, já que o uso contínuo e prolongado desses pode causar adversidades como a sensibilidade nos dentes. O acompanhamento de um odontólogo no tratamento evita os efeitos indesejados, priorizando a saúde do paciente e o seu objetivo, de forma que a eficácia do clareamento seja maior e seja mantida sua integridade.

Se o objetivo do paciente for o clareamento propriamente dito, é necessária a busca por outros produtos com maior eficácia, que devem ser prescritos pelo profissional da odontologia, pois só ele deve decidir por quanto tempo deverá persistir o tratamento e indicar o clareamento dentário apropriado para cada paciente, já que as técnicas para esse efeito são individualizadas, ou seja, nem todas funcionam em todos os pacientes ou até mesmo em todos os dentes ${ }^{51}$.

\section{Considerações finais}

Diante dos estudos realizados com base na eficácia dos produtos de autocuidado para clareamento dental e com base na revisão de literatura, nota-se que esses não substituem o tratamento cla- reador convencional em consultório e a técnica do clareamento caseiro supervisionado pelo dentista, visto que a maioria não cumpre sua ação clareadora. Cabe ao dentista propor ao paciente a técnica mais segura e eficaz de clareamento para seu caso, sendo de extrema importância o acompanhamento do profissional.

É necessário que mais estudos sejam realizados, a fim de gerar mais informações ao paciente quanto à segurança dos produtos clareadores de autocuidado assim como provas da eficácia desses.

\section{Abstract}

\begin{abstract}
Objective: To perform a literature review on the various methods of self-care products for tooth whitening, seeking to assess evidences regarding their effectiveness and limitations. Literature review: The concern with dental aesthetics has gained importance in recent years, and at-home tooth whitening has been an increasingly sought treatment in the dental office. Home treatment with trays containing bleaching agents have shown satisfactory results with few adverse effects, and it is considered the gold standard for treating vital teeth. With the success of this technique, new products have appeared on the market, which are freely traded and could be used without the supervision of a dentist; these products are known as self-care products for tooth whitening. Different products may be found within this classification (bleaching strips, bleaching toothpastes, rinses, varnishes), but the relatively recent time of their introduction and the small number of clinical studies performed raises doubts about their effectiveness and potential adverse effects. The adhesive strip showed satisfactory results in some researches, besides being practical and simple to use. Final considerations: From the studies based on the effectiveness of self-care products for tooth whitening and based on the literature review, it is noted that these products do not replace a conventional in-office bleaching treatment, since most do not fulfill their whitening action.
\end{abstract}

Keywords: Tooth bleaching. Color. Dentifrices.

\section{Referências}

1. Francci C, Marson FB, Briso ALF, Gomes MN. Dental Bleaching-Current Concepts and Techniques. Rev Assoc Paul Cir Dent 2010; (1):78-89.

2. Demarco FF, Meirelles SS, Masotti AS. Over-The-Counter Whitening Agents: A Concise Review. Braz Oral Res 2009; 23(Spec Iss I):64-70.

3. American Dental Association (ADA). Acceptance Program Guidelines. Dentist dispensed home-use tooth bleaching products 2006; 1-14.

4. Aschill TM, Hellwig E, Schmidale S, Sculean A, Arweiler NB. Efficacy, side-effects and patients' acceptance of different bleaching techniques (OTC, in-office, at-home). Oper Dent 2005; 30(2):156-63.

5. Garone FW, Abreu e Silva V. Lesões não cariosas. São Paulo: Livraria e Editora Santos; 2008. 
6. Anton ARS, Lima MJP, Araújo RPC. Dentifrício peróxido de hidrogênio: ação clareadora? Rev Odonto Ciência 2009; 24(2):161-7.

7. Silva JPFL, Netto MCP, Guimarães RP, Silva CHV. Avaliação da eficácia de cremes dentais clareadores com uso associado ou não de escova dental especial. RFO De Lins 2011; 21(2):31-9.

8. Walsh TF, Rawlinson A, Wildgoose D, Marlow I, Haywood J, Ward JM. Clinical evaluation of the stain removing ability of a whitening dentifrice and stain controlling system. J Dent 2005; 33:413-8.

9. Gerlach RW, Barker ML. Clinical response of three directto consumer whitening products: strips, paint-on gel, and dentifrice. Compend Cont Educ Dent 2003; 24:458-65.

10. Magalhaes AC, Moron BM, Comar LP, Buzalaf MAR. Uso racional dos dentifrícios. RGO 2011; 59:615-25.

11. Mello DCCL. Avaliação in vitro do poder clareador doa cremes dentais sobre a estrutura dental [Trabalho de Conclusão de Curso - Graduação em Odontologia]. Campina Grande: Universidade Estadual da Paraíba; 2014.

12. Thiesen $\mathrm{CH}$. A influência de dentifrícios com agente dessensibilizante na dor induzida por clareamento dental de consultório [Dissertação de Mestrado]. Santa Catarina: Universidade Federal de Santa Catarina; 2012.

13. Pontes ECV, Gomes LRM, Soares DGS, Ribeiro APD, Hebling J, Costa CAS. Citotoxicidadetransamelodentinária de uma fita clareadora com $10 \%$ de peróxido de hidrogênio sobre células de linhagem odontoblástica. Rev Odontol Bras Central 2012; 21(56):376-82.

14. D'Altoé LF. Estudo comparativo entre o sistema clareador de tiras e a técnica de clareamento caseiro supervisionado [Trabalho de Especialização em Dentística Restauradora]. Florianópolis: Escola de Aperfeiçoamento Profissional da Associação Brasileira de Odontologia; 2003.

15. Ernest CP, Marroquin BB, Willershausen-Zönnchen B. Effects of hydrogen peroxide-containing bleaching agents on the morphology of human enamel. Quintessence Int 1996; 27:53-6.

16. Ramos AB, Monnerat AF, Perez CR. Avaliação da eficácia das fitas para clareamento dental. Rev Bras Odontol 2014; 71(2):198-202.

17. Jabôr NM. Clareamento de dentes vitais com tiras clareadoras, vernizes clareadores e Led [Trabalho de Conclusão de Curso - Graduação em Odontologia]. Florianópolis: Universidade Federal de Santa Catarina; 2005.

18. Brunato MC. Clareamento caseiro em dentes vitais supervisionado pelo dentista versus não supervisionado [Trabalho de Conclusão de Especialização em Dentística]. Florianópolis: Escola de Aperfeiçoamento Profissional da Associação Brasileira de Odontologia; 2005.

19. Conselho Regional de Odontologia do Rio Grande do Norte. Resolução da Anvisa determina que clareador dental só poderá ser vendido com receita de dentista. 2016 [citado 2016 abr. 2]. Disponível em URL: http://www.crorn.org.br/ noticias/ver/705.

20. Agência Nacional de Vigilância Sanitária. Proposta de resolução RDC que dispõe sobre o controle de agentes clareadores dentais. 2014 [citado 2016 abr. 2]. Disponível em URL: http://portal.anvisa.gov.br/wps/wcm/connect/5cd2a70043 6e3db0b586bdfbb3e9d9ad/Proposta+de+CP_Clareadores. pdf?MOD=AJPERES.

21. Henn S, Lima GS, Massoti AS, Demarco FF. Home bleaching using whitening strips: a case report. Clín Int J Braz Dent 2010; 6(3):300-6.

22. Consolaro A. Fitas adesivas, "Vanish" e técnica clareadora caseira: aspectos importantes a serem considerados. Rev Dental Press Estét 2013; 10(1):58-63.
23. Medeiros CSEA. Avaliação da técnica de clareamento profissional com e sem fotoativação [Trabalho de Conclusão de Curso - Graduação em Odontologia]. Amazonas: Faculdade de Odontologia da Universidade Federal do Amazonas; 2011.

24. Lima FG, Rotta TA, Penso S, Meireles SS, Demarco FF. In vitro evaluation of the whitening effect of mouth rinses containing hydrogen peroxide. Braz Oral Res 2012; 26(3):269-74.

25. Johnson \& Johnson do Brasil Indústria e Comércio de Produtos para Saúde Ltda. LISTERINE Whitening PréEscovação. 2016 [citado 2016 abr. 2]. Disponível em URL: https://www.listerine.com.br/produtos/listerine-whitening-pre-escovacao.

26. Colgate-Palmolive Company. Colgate Luminous White. 2016 [citado 2016 abr. 2]. Disponível em URL: http://www. colgate.com.br/app/Luminous/BR/produtos.cvsp.

27. Procter \& Gamble do Brasil. Antisséptico Bucal 3D WHITE. 2015 [citado 2016 abr. 2]. Disponível em URL: http:// www.oralb.com/brazil/produtos/enxaguantes-bucais/3d-white/\#overview-0.

28. Jhingta P, Bhardwaj A, Sharma D, Kumar N, Bhardwaj VK, Vaid S. Effect of hydrogen peroxide mouthwash as an adjunct to chlorhexidine on stains and plaque. J Indian Socperiodontol 2013; 17(4):449-53.

29. Hasturk H, Nunn M, Warbington M, Van Dyke TE. Efficacy of a fluoridated hydrogen peroxide-based mouthrinse for the treatment of gingivitis: a randomized clinical trial. J Periodontol 2004; 75(1):57-65.

30. Torres CRG, Perote LCCC, Gutierrez NC, Pucci CR, Borges AB. Efficacy of mouth rinses and toothpaste on tooth whitening. Oper Dent 2013; 38(1):57-62.

31. Silva TPT. Avaliação in vitro do efeito de agentes clareadores na microdureza do esmalte dental [Dissertação de Pós-Graduação em Farmacologia]. Fortaleza: Faculdade de Medicina da Universidade Federal do Ceará; 2013.

32. Consolaro A, Francischone LA, Consolaro RB. O clareador dentário atua como cocarcinógeno na mucosa bucal, inclusive quando em dentifrícios e antissépticos: Fundamentos para orientação de pacientes ortodônticos e como evitar seus efeitos indesejáveis. Dental Press J Orthod 2011; 6(2):28-35.

33. Worschech CC. Brushing effect of abrasive dentifrices during at-home with $10 \%$ carbamide peroxide on enamel surface roughness. J Contemp Dent Pract 2006; 15(7):25-34.

34. Slezak B, Santarpia P, Xu T, Monsul-Barnes V, Heu R, Stranick $\mathrm{M}$ et al. Perfil de segurança de um novo gel líquido branqueador. Educ Dent 2002; 4-11.

35. Maia EAV, Vieira LCC, Baratieri LN, Andrade CA. Clareamento em dentes vitais: estágio atual. Clin Int J Braz Dent 2005; 1(1):8-19.

36. Nathoo S, Petrone E, Devizio W, Chaknis P, Volpe AR. A six week clinical study to compare the stain removal efficacy of three dentifrices. J Clin Dent 2002; 13(2):91-4.

37. Gerlach RW, Barker ML, Tucker HL. Clinical response of three whitening products having different peroxide delivery: comparison of tray, paint-on gel, and dentifrice. J Clin Dent 2004; 15(4):112-7.

38. Kishta-Derani M, Neiva G, Yaman P, Dennison D. In vitro evaluation of tooth-color change using four paint-on tooth whiteners. Oper Dent 2007; 32(4):394-8.

39. Reichelt Ely AL. Agentes clareadores dentais: estudo comparativo entre formas farmacêuticas e técnicas de utilização [Monografia]. Porto Alegre: Universidade Federal do Rio Grande do Sul; 2010.

40. Brasil. Lei $\mathrm{n}^{\circ}$ 6.360/1976. [citado 2016 abr. 3]. Disponível em URL: http://www.jusbrasil.com.br/topicos/11327826/paragrafo-1-artigo-58-da-lei-n-6360-de-23-de-setembro-de-1976. 
41. Oliveira WHT, Prado MM, Silva RF. Publicidade odontológica na internet: os sites de compra coletiva. RBOL 2014; 1(1):78-91.

42. Peixe Urbano Web Serviços Digitais, LTDA. Creme dental. 2015 [citado2016abr. 3]. DisponívelemURL:https://www.peixeurbano.com.br/pelotas/busca? $\mathrm{q}=$ creme+dental+clareador.

43. Buscapé Company Informação e Tecnologia Ltda. Creme dental. 2016 [citado 2016 abr. 3]. Disponível em URL: http:// www.buscape.com.br/creme-dental.

44. Peixe Urbano Web Serviços Digitais, LTDA. Fita clareadora. 2015 [citado 2016 abr. 3]. Disponível em URL: https://www. peixeurbano.com.br/pelotas/busca?q=fita+clareadora

45. Peixe Urbano Web Serviços Digitais, LTDA. Simply White e Simply White Night. 2015 [citado 2016 abr. 3]. Disponível em URL: https://www.peixeurbano.com.br/pelotas/busca?q= Simply+Whinte+e+Simply+White+Night.

46. PeixeUrbano Web Serviços Digitais,LTDA. Crest NightEffects. 2015 [citado 2016 abr. 3]. Disponível em URL: https://www. peixeurbano.com.br/pelotas/busca?q=Crest+Night+Effects+.

47. Brasil. Lei $n^{\circ} 8.078$, de 11 de setembro de 1990. Dispõe sobre a proteção do consumidor e dá outras providências. Brasília, DF, 1990.

48. Brasil. Lei $\mathrm{n}^{\circ}$ 5.081, de 24 de agosto de 1966. Regula o exercício da Odontologia. Brasília, DF, 1966.

49. Conselho Federal de Odontologia. Resolução de no 118 , de 11 de maio de 2012. Código de Ética Odontológica.

50. Hoic D, Dixit N, Prencipe M, Subramanyam R, Cameron R, Malak RA, et al. The technology behind Colgate Simply White Toothpaste. J Clin Dent 2004; 15(2):37-40.

51. Lizarelli RFZ. Clareamento caseiro. RGO Porto Alegre 1994; 5(42):275-8

\section{Endereço para correspondência:}

Ana Carolina Gluszevicz

Faculdade de Odontologia

Universidade Federal de Pelotas

Avenida J. K. de Oliveira, 2200

96080-000, Pelotas, RS

Telefone: (53) 8108-0041/ (53) 8119-1056

E-mail: maria.mmendes@hotmail.com

Recebido: 13/08/15. Aceito: 23/05/16. 